\title{
Matrix Metalloproteinases 2 and 9 Immunoexpression in Prostate Carcinoma at the Positive Margin of Radical Prostatectomy Specimens
}

\author{
Romano Oguić, ${ }^{1}$ Vladimir Mozetič, ${ }^{2}$ Eleonora Cini Tešar, ${ }^{3}$ Dora Fučkar Čupić, ${ }^{4}$ \\ Elvira Mustać, ${ }^{4}$ and Gordana Đorđević ${ }^{4}$ \\ ${ }^{1}$ Department of Urology, Clinical Hospital Centre Rijeka, Krešimirova 42, 51000 Rijeka, Croatia \\ ${ }^{2}$ Medico Polyclinic, Agatićeva 8, 51000 Rijeka, Croatia \\ ${ }^{3}$ Department of Oncology, Clinical Hospital Centre Rijeka, Krešimirova 42, 51000 Rijeka, Croatia \\ ${ }^{4}$ Department of Pathology, School of Medicine, University of Rijeka, Braće Branchetta 20, 51000 Rijeka, Croatia
}

Correspondence should be addressed to Gordana Đorđević; gordana.dordevic@medri.uniri.hr

Received 15 February 2014; Accepted 5 June 2014; Published 6 July 2014

Academic Editor: Shahid Pervez

Copyright (C) 2014 Romano Oguić et al. This is an open access article distributed under the Creative Commons Attribution License, which permits unrestricted use, distribution, and reproduction in any medium, provided the original work is properly cited.

\begin{abstract}
The aim of this study was to evaluate the expression of matrix metalloproteinase 2 (MMP-2) and matrix metalloproteinase 9 (MMP9) in prostate cancer in the main tumor mass and tumor cells at the positive margin as well as the influence of these biomarkers on the biochemical recurrence of the disease in prostatectomy patients. Tissue microarrays of 120 archival prostate carcinoma samples were immunohistochemically evaluated for MMP-2 and MMP-9 expression and compared with clinicopathological parameters. Tumors with positive surgical margins showed significantly higher overall expression of MMP-9 versus tumors with negative resection margins $(P=0.0121)$. MMP-9 expression was significantly elevated in tumors from patients who had biochemical recurrence $(P=0.0207)$. In the group of patients with negative margins, MMP-9 expression above the cut-off value was significantly associated with recurrence $(P=0.0065)$. Multivariate analysis indicated that MMP-9 is a good predictor of biochemical recurrence (odds ratio $=10.29 ; P=0.0052$ ). Expression of MMP-2 in tumor cells was significantly higher at the positive margins than in the main tumor mass $(P=0.0301)$. The present results highlight the potential value of MMP-2 and MMP-9 expression for predicting the behavior of prostate tumors after prostatectomy with both positive and negative surgical margins.
\end{abstract}

\section{Introduction}

Extensive studies of the matrix metalloproteinases (MMPs) indicate that a large number of MMP family factors play active roles in carcinogenesis and metastasis [1-3]. Many studies have focused on the role of MMPs and their inhibitors in the prognoses of various types of malignancies. Most published data on MMP-2 and MMP-9 address the role of these proteins in encouraging the aggressiveness of cancers. Increased expression of MMP-2 correlates with low survival in patients with breast cancer [4-8], is associated with a 4.5fold higher relative risk of mortality from skin melanoma, and predicts the risk of metastasis in uveal melanoma $[9,10]$. Increased expression of MMP-2 is a sign of poor prognosis in cancer of the stomach and pancreas [11-13] as well as the prostate [14].

Kuvaja et al. concluded that low levels of serum proMMP-2 correlate with aggressive cancer behavior [15]. High MMP-2 expression in hematological malignancies suggests good prognosis; in contrast, high MMP-9 expression is a sign of poor disease outcome [16]. The function of MMP-9 is still controversial in solid tumors. Scorilas et al. proposed that MMP-9 immunoreactive protein can be a favorable sign for node-negative breast cancer [17]. On the other hand, investigations of high MMP-9 levels in plasma or serum samples showed that MMP-9 expression may be associated with increased risk of recurrence and poor prognosis $[18,19]$. In prostate carcinoma, MMP-2 and MMP-9 are 
novel molecular biomarkers that reflect the invasive and metastatic potential of this type of carcinoma [20]. The standard biomarkers that significantly predict the clinical and biochemical recurrence of prostate cancer are preoperative serum prostate-specific antigen (PSA), pathological grade according to Gleason score, positive surgical margins (with and without extraprostatic extension), and capsular incision adding up the following factors as of prognostic significance: presence of perineural, angiolymphatic, seminal vesicle invasion and extraprostatic tissue invasion. However, the real prognostic importance of positive margins remains to be defined. Few studies have emphasized the behavior of the previously mentioned biomarkers on the positive edge of the resection in comparison to their behavior in the bulk tumor mass. Recently, Cao et al. addressed the issue of positive resection margins in radical prostatectomy and found that the Gleason score at the edge of the tumor resection is predictive of biochemical recurrence [21]. There are currently no studies on the predictive value of MMP expression on the edge of the resection in radical prostatectomy.

The aim of this study was to measure the expression levels of MMP- 2 and MMP-9 in the main tumor mass and in tumor cells on the positive margin and to compare these expression levels with Gleason score and tumor size in patients treated by radical prostatectomy. We also examined the influence of these biomarkers on biochemical recurrence of the disease in prostatectomy patients.

\section{Methods}

2.1. Patients. During the period from 2001 to 2006, acinar adenocarcinoma was discovered by ultrasound-guided biopsy of the prostate in 793 patients at the Clinic of Urology, Clinical Hospital Center Rijeka, Croatia. In accordance with the criteria from the European Urological Association, candidates for radical prostatectomy had a Gleason score of 7 or less, PSA levels below $10 \mathrm{ng} / \mathrm{mL}$, and clinically confirmed prostate cancer in stage T1c/T2c (normal preoperative computed tomography findings and pelvic bone scintigraphy) [22]. From 2001 to 2006, 120 patients who had undergone radical prostatectomy were chosen from medical documentation files and adequate clinical data were collected. Two groups of patients were formed: a group of 71 patients having tumors with negative surgical margins and a second group of 49 patients with tumors showing positive surgical margins. Tumor material, obtained from radical prostatectomy, was selected for the construction of tissue microarrays. Representative areas on the hematoxylin and eosin-stained sections were carefully selected and marked on the corresponding paraffin blocks. From each carcinoma, two tissue cores ( $1 \mathrm{~mm}$ in diameter) were obtained from the main tumor mass and two cores were taken from the tumor at the positive surgical margin; these samples were arrayed in a recipient paraffin block using MTA Booster OI Manual Tissue Arrayer (Alphalyse, Plaisir, France).

2.2. Immunohistochemistry. Immunohistochemical staining for MMP-2 (17B11 mouse monoclonal antibody, Vision Biosystems Novocastra, Newcastle, UK) and MMP-9 (17W2
TABLE 1: Clinical and pathological features of patients with prostate cancer treated with prostatectomy.

\begin{tabular}{lc}
\hline Feature & \\
\hline Age of patients (years, median) & 64.12 \\
Preoperative serum PSA (ng/mL, median) & 8.50 \\
Tumor size (cm, median) & 2.25 \\
Pathologic stage (\%) & \\
pT2 & 74.00 \\
pT3 & 26.00 \\
Gleason score (\%) & \\
$\quad \leq 6$ & 65.40 \\
$\quad \geq 7$ & 34.60 \\
Margin status (\%) & \\
$\quad$ Positive & 58.82 \\
$\quad$ Negative & 41.17 \\
Disease-free survival (months) & \\
$\quad$ Negative margin group & 31.2 \\
$\quad$ Positive margin group & 22.6 \\
\hline
\end{tabular}

mouse monoclonal antibody NCL-MMP-9-493, Vision Biosystems Novocastra) was carried out with an automated immunostainer (OptiMax Plus, BioGenex) employing a standard biotin-streptavidin method. Heat-induced pretreatment for antigen retrieval (slides were immersed in a $10 \mathrm{mM}$ citrated buffer, $\mathrm{pH} 6.0$, at $95^{\circ} \mathrm{C}$ for $5 \mathrm{~min}$ ) was carried out prior to incubation with primary antibody; the Dako En/Vision+/HRP Kit was used to visualize MMP expression. Intestinal tissue of patients with inflammatory bowel disease (as recommended by the manufacturer) and the positive control for MMP-9 were normal liver tissues (as recommended by the manufacturer).

The expression level of each protein was determined with image analysis system ISSA 3.1 software (Zagreb, Croatia). Staining was evaluated as the percentage of cytoplasmicpositive tumor cells multiplied by the staining intensity; this value was expressed as a histoscore. Clinicopathological data obtained from patient medical records included age, tumor size, TNM stage, Gleason score, and margin status of the prostatectomy specimens (Table 1). We separately examined the immunoexpression of the MMPs relative to localization within the tumor mass (MMP expression on the positive surgical margins and in the bulk of the tumor mass).

\section{Results}

We found a mean Gleason score significantly higher in the group with positive surgical margins than in the group with negative surgical margins $(P=0.0032$; Figure 1$)$.

Immunohistochemical staining against MMP-2 and MMP-9 in glands with hyperplastic epithelium indicated weak or negative expression of these proteins, except for glands with high-grade PIN, where the expression was stronger (Figures 1). MMP-2 and MMP-9 immunoexpression in tumor cells was cytoplasmic, finely granular, and varied in intensity and percentage. We did not find tumors that were negative for the expression of these proteins (Figures 1). 
TABLE 2: Expression of MMP-2 and MMP-9 in the main tumor mass and on the resection margin.

\begin{tabular}{|c|c|c|c|}
\hline & $N$ & Average & $P$ \\
\hline \multicolumn{4}{|l|}{ MMP-2 } \\
\hline Tumor & 95 & 98.9160 & \multirow[t]{2}{*}{0.0301} \\
\hline Margin & 43 & 109.0237 & \\
\hline Tumor mass, positive surgical margin & 43 & 91.3581 & \multirow[t]{2}{*}{0.1833} \\
\hline Tumor mass, negative surgical margin & 52 & 105.1658 & \\
\hline \multicolumn{4}{|l|}{ MMP-9 } \\
\hline Tumor & 106 & 142.1780 & \multirow[t]{2}{*}{0.1214} \\
\hline Margin & 45 & 171.0924 & \\
\hline Tumor mass, positive surgical margin & 45 & 157.2962 & 0.0121 \\
\hline Tumor mass, negative surgical margin & 61 & 131.0252 & \\
\hline
\end{tabular}

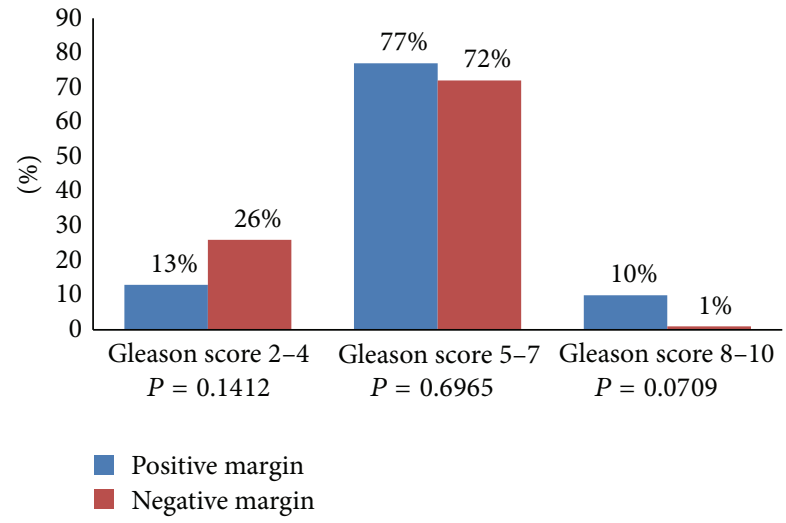

FIGURE 1: Analysis of Gleason score in patients with positive or negative surgical margins.

MMP-2 was expressed not only in the cytoplasm of tumor cells but also in the cytoplasm of prostatic stromal cells, endothelium, fibroblasts, smooth muscle cells, macrophages, and lymphocytes. The average histoscore of MMP-2 expression per tumor was 98.9160 (standard deviation (SD) 50.1750). MMP-2 was significantly more strongly expressed in tumor cells on the positive margins than in the main tumor mass, with an average of 109.0237 (SD 64.0751; $P=0.0301)$. In the stroma, the strongest MMP-2 expression occurred in the cytoplasm of inflammatory cells, especially macrophages (Figure 3).

MMP-9 expression was detected in the cytoplasm of tumor cells, while stromal cells were weakly positive for this marker; staining could be categorized as cytoplasmic or nuclear plus cytoplasmic. The cytoplasm of some tumors contained more pronounced luminal expression of MMP-9, while, in other tumors, this staining was diffuse or mostly peripheral. The average amount of MMP-9 immunostaining was higher than that of MMP-2 in both examined locations. The mean MMP-9 expression was 142.1780 (SD 51.0358) in the main tumor mass and 171.0924 (SD 61.9203) at the positive margin of resection. Although there was no significant difference in MMP-9 immunoexpression between the main tumor mass and the edge, a group of tumors
TABLE 3: MMP-2 and MMP-9 expression in terms of total Gleason score and tumor stage and size.

\begin{tabular}{lcc}
\hline & Average histoscore & $P$ \\
\hline MMP-2 expression in margin & & \\
Gleason $<7$ & 98.103 & 0.7417 \\
Gleason $\geq 7$ & 127.453 & \\
Stage 2 & 113.390 & 0.5867 \\
Stage 3 & 102.089 & \\
MMP-2 expression in tumor & & \\
Gleason $<7$ & 99.121 & 0.9389 \\
Gleason $\geq 7$ & 98.146 & \\
Stage 2 & 94.942 & 0.1712 \\
Stage 3 & 110.771 & \\
Size $\leq 1 \mathrm{~cm}$ & 114.165 & 0.5207 \\
Size $>1 \mathrm{~cm}$ & 97.342 & \\
MMP-9 expression in margin & & \\
Gleason $<7$ & 173.391 & 0.7417 \\
Gleason $\geq 7$ & 166.927 & \\
Stage 2 & 173.116 & 0.9093 \\
Stage 3 & 170.875 & \\
MMP-9 expression in tumor & & 0.0378 \\
Gleason $<7$ & 139.472 & \\
Gleason $\geq 7$ & 153.812 & \\
Stage 2 & 137.432 & \\
Stage 3 & 155.930 & \\
Size $\leq 1 \mathrm{~cm}$ & 95.000 & \\
Size $>1 \mathrm{~cm}$ & 143.067 & \\
\hline
\end{tabular}

with positive surgical margins showed significantly stronger overall expression of MMP-9 compared to a group of tumors with negative resection margins $(P=0.0121$; Table 2$)$.

Increased expression of MMP-2 and MMP-9 was found in tumors of better differentiation and higher Gleason score but with no significant differences between tumors with Gleason score $<7$ and high-grade cancers with Gleason score $\geq 7$. Regarding disease stage, MMP-2 staining was slightly stronger in higher-stage tumors but without statistical significance. Tumor size was significantly associated with MMP-9 
TABLE 4: ROC analysis.

\begin{tabular}{lclll}
\hline & & AUC & 95\% confidence interval & $P$ \\
\hline MMP-2, margin & All & 0.589 & 0.413 to 0.749 & 0.3596 \\
\hline \multirow{2}{*}{ MMP-2, tumor mass } & All & 0.524 & 0.408 to 0.638 & 0.7610 \\
& Negative margin & 0.525 & 0.352 to 0.694 & 0.8108 \\
& Positive margin & 0.693 & 0.532 to 0.826 & 0.0988 \\
\hline MMP-9, margin & All & 0.551 & 0.379 to 0.715 & 0.6352 \\
\hline \multirow{2}{*}{ MMP-9, tumor mass } & All & 0.626 & 0.515 to 0.728 & 0.0582 \\
& Negative margin & 0.513 & 0.343 to 0.680 & 0.8970 \\
\hline
\end{tabular}

TABLE 5: MMP-2 and MMP-9 expression and recurrence regarding cut-off values determined by the Youden index.

\begin{tabular}{ccccccccc}
\hline & & Youden index & 95\% CI & Associated criterion & Sensitivity & 95\% CI & Specificity & 95\% CI \\
\hline MMP-2 & all margins & 0.2609 & 0.1405 to 0.3344 & $>52.5$ & 100.00 & 75.3 to 100.0 & 26.09 & 10.2 to 48.4 \\
\hline \multirow{2}{*}{ MMP-2 TM } & all & 0.1679 & 0.1203 to 0.1839 & $>158.33$ & 23.81 & 8.2 to 47.2 & 92.98 & 83.0 to 98.1 \\
& neg. margin & 0.2475 & 0.1605 to 0.2910 & $\leq 90$ & 76.92 & 46.2 to 95.0 & 47.83 & 26.8 to 69.4 \\
& pos. margin & 0.3456 & 0.1471 to 0.4632 & $>90$ & 87.50 & 47.3 to 99.7 & 47.06 & 29.8 to 64.9 \\
\hline MMP-9 & all margins & 0.1827 & 0.1314 to 0.189 & $\leq 115$ & 30.77 & 9.1 to 61.4 & 87.50 & 67.6 to 97.3 \\
\hline \multirow{2}{*}{ MMP-9 TM } & all & 0.2553 & 0.1242 to 0.3941 & $>141.67$ & 65.22 & 42.7 to 83.6 & 60.32 & 47.2 to 72.4 \\
& neg. margin & 0.1731 & 0.1314 to 0.1795 & $>195$ & 7.69 & 0.2 to 36.0 & 75.00 & 53.3 to 90.2 \\
& pos. margin & 0.3974 & 0.1729 to 0.5718 & $>175$ & 50.00 & 18.7 to 81.3 & 89.74 & 75.8 to 97.1 \\
\hline
\end{tabular}

CI: confidence interval; TM: tumor mass.

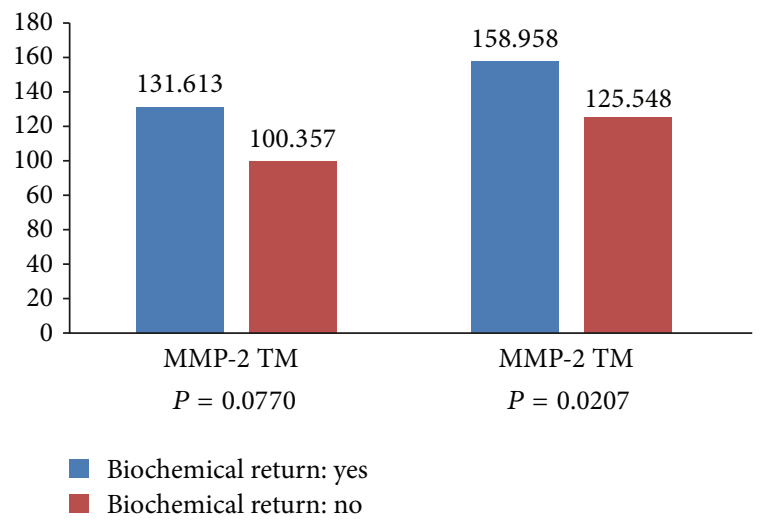

FIGURE 2: Biochemical recurrence with respect to molecular biomarkers in patients with tumors with negative surgical margins.

expression, which was high in tumors larger than $1 \mathrm{~cm}(P=$ 0.038 ; Table 3 ).

We analyzed the biochemical recurrence of the disease in relation to MMP expression in the group of patients with negative surgical margins that did not receive any therapy after prostatectomy that could have affected the outcome. MMP-9 expression was significantly elevated in tumors from patients who experienced biochemical recurrence $(P=$ 0.0207). MMP-2 expression in tumors tended to be increased in patients with biochemical recurrence $(P=0.0770$; Figure 2).

We used a receiver operating characteristic (ROC) analysis, which is widely recognized as a measure of a diagnostic test's discriminatory power. The maximum value for the area under the curve (AUC) is 1.0, indicating a (theoretically) perfect test (100\% sensitive and $100 \%$ specific). An AUC value of 0.5 indicates no discriminative value. We evaluated MMP-2 and MMP-9 expression as predictors of recurrence in patients with tumors with negative surgical margins. MMP-9 expression was detected in all patients (Table 4). Furthermore, cut-off values for these parameters and groups were determined based on the Youden index (Table 5).

Analysis of the proportions of patients with and without recurrence in terms of increased MMP-9 expression revealed significant differences between the two groups at a significance level of $0.1(P=0.0635)$.

In the group of patients with negative margins, increased MMP-9 expression above the cut-off value of 175 was significantly associated with recurrence $(P=0.0065)$, while MMP-2 expression was not (Table 6).

Univariate and multivariate analysis showed that MMP9 expression is a good predictor of biochemical recurrence (Table 7).

Recurrence-free survival was not significantly shorter in the group of patients with tumors with negative margins and high MMP-9 expression (Kaplan-Meier analysis, $P=$ 0.5555).

\section{Discussion}

Prostate diseases are often present in the older population. Benign enlargement or inflammation of the prostate affect quality of life, while prostate cancer can be life threatening. Unfortunately, the routine diagnostic triad of a combination 


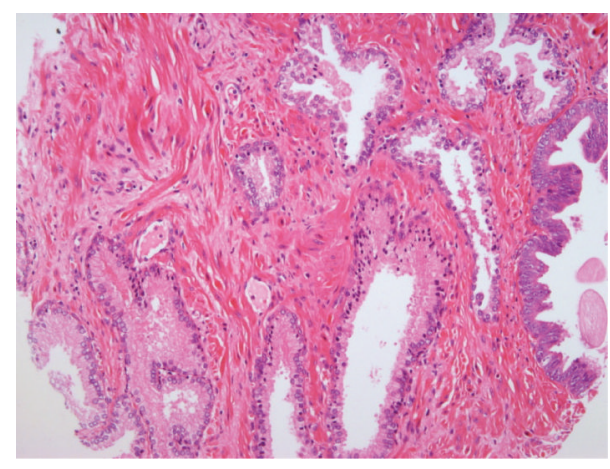

(a)

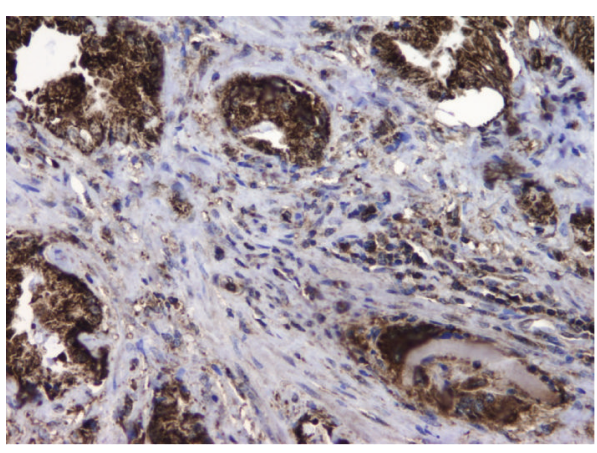

(c)

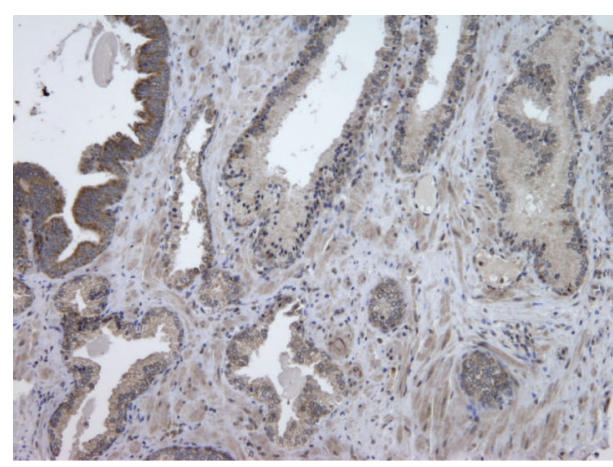

(b)

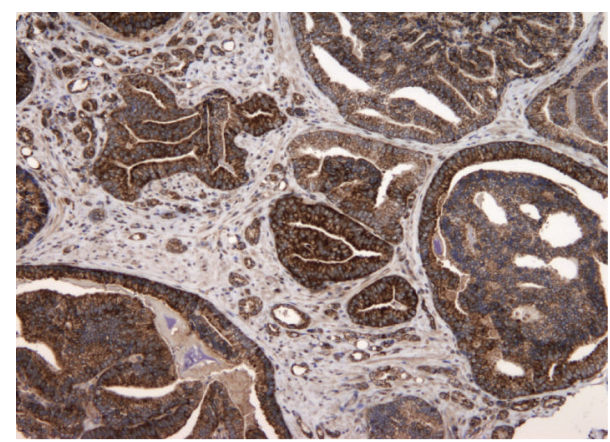

(d)

Figure 3: (a) Glands lined with hyperplastic epithelium and PIN (hematoxylin and eosin staining, 100x). (b) MMP-2 and MMP-9 immunohistochemistry indicates weak or negative expression in hyperplastic epithelium, except for the PIN, where the expression is strong (100x). (c) MMP-9 immunoexpression in moderately differentiated prostate cancer. Note the intense staining in the cytoplasm of tumor cells and the strongly positive reaction in the cytoplasm of stromal cells (200x). (d) MMP-2 immunoexpression in poorly differentiated prostate cancer. Note the strong cytoplasmic staining intensity (200x).

TABLE 6: MMP-2 and MMP-9 expression in terms of cut-off value in tumors with negative margins.

\begin{tabular}{lcccc}
\hline \multicolumn{2}{c}{ Tumors with negative margins } & \multicolumn{3}{c}{ Recurrence } \\
& & $N$ & Yes & No \\
\hline \multirow{2}{*}{ MMP-9, tumor margin } & $0(\leq 175)$ & $35(90 \%)$ & $5(10 \%)$ & 39 \\
& $1(>175)$ & $4(44 \%)$ & $5(56 \%)$ & 9 \\
$P$ & & 0.0065 & & \\
\hline \multirow{2}{*}{ MMP-2, tumor margin } & $0(\leq 90)$ & $16(94 \%)$ & $1(6 \%)$ & 17 \\
& $1(>90)$ & $18(72 \%)$ & $7(28 \%)$ & 25 \\
$P$ & & 0.1674 & & \\
\hline
\end{tabular}

of an abnormal PSA level, digital rectal examination, and transrectal sonography-guided biopsy of the prostate does not always result in detection of the tumor (sensitivity $75 \%$, specificity 68\%). If one or more tests are abnormal, prostate biopsy must be performed to confirm or exclude malignant disease. For now, the best information about limitations, disease stage, and the malignant potential of the prostate cancer are obtained by histopathological examination.

Pathological classification of prostate cancer after radical prostatectomy provides important prognostic information, and an accurate histopathological report is thought to be fundamental for treating and monitoring patients. Preoperative serum PSA levels, pathological grade according to
Gleason score, invasion into the seminal vesicle, positive surgical margins with and without extraprostatic extension, and capsular incision are significant predictors of clinical and biochemical recurrence. The real prognostic significance of positive margins and capsule incision remains to be defined $[23,24]$.

Approximately $5-43 \%$ of patients with radical prostatectomy have positive surgical margins, and only $25-47 \%$ of them develop biochemical recurrence [25]. These observations suggest the utility of further subclassification of positive margins in order to identify patients with higher risks of recurrence. In our study, the overall rate of positive surgical margins was $29.8 \%$ in pT3 tumors and up to $90 \%$ in other tumors, which is similar to other studies [26-28].

The time to recurrence, regardless of treatment, was significantly shorter in patients with locally confined tumors with positive surgical margins. This observation is consistent with the results of Bostwick et al., who reported a positive impact of positive prostatectomy margins on biochemical recurrence of the disease [29]. Few studies have emphasized the potential utilities of biomarker expression on positive surgical margins relative to the main tumor mass. In our study, which used tissue microarrays, the average Gleason score was significantly higher in the group of patients with tumors with positive margins, partially confirming the results of Cao et al. [30]. There was no significant difference between 
TABLE 7: Analysis of the significance of MMP-9 expression for predicting biochemical recurrence.

\begin{tabular}{lccccc}
\hline & & Univariate analysis & \multicolumn{3}{c}{ Multivariate analysis } \\
& OR & $95 \%$ CI & $P$ & OR & $95 \%$ CI \\
\hline MMP-2, TM & 6.2222 & 0.6888 to 56.2057 & 0.1035 & 5.1721 & 0.4924 to 54.3318 \\
MMP-9, TM & 8.7500 & 1.7411 to 43.9746 & 0.0078 & 10.2909 & 1.6284 to 65.0367 \\
& & & & & $P=0.0052$ \\
\hline
\end{tabular}

OR: odds ratio; CI: confidence interval; and TM: tumor mass.

the average Gleason score on the margins of the tumor resection and the average Gleason score in the main tumor mass; this observation may be due to the strict selection of patients for prostatectomy, which usually requires Gleason scores $\leq 7$.

The human family of MMPs consists of at least 24 proteinases involved in degradation of the extracellular matrix and components of the basement membrane. The MMPs are directly involved in the essential cellular processes of proliferation, differentiation, angiogenesis, and apoptosis through their ability to catalyze the hydrolysis of various substrates, including the precursors of cytokines, growth factors, and hormone receptors. A number of studies revealed positive correlations between tumor invasion and the activities of the gelatinases MMP-2 and MMP-9. Expression of these MMPs is most frequently associated with tumor aggressiveness and overall survival, and they are used as markers of malignant phenotypes [31-33].

In prostate cancer, MMP-2 and MMP-9 expression indicate the tumor's invasive and metastatic potential [34]. Given the development of specific MMP inhibitors, we assumed that the immunohistochemical determination of the expression of these two MMPs would be of particular importance for therapy, especially if it indicated whether the tumor was at high risk of recurrence. In our study, we sought to determine whether MMP-2 and/or MMP-9 expression differed between aggressive cancers on the positive margin of the resection that progress and cancers that remain localized. We detected a significant difference in MMP-2 expression on the positive margin versus that in the main tumor mass $(P=0.0301)$. MMP-9 expression at the margin of the resection did not significantly differ from the expression in the main tumor mass, but, in the group of tumors with positive margins, MMP-9 was significantly more strongly expressed overall versus the group of tumors with negative resection margins $(P=0.0121)$.

In the group of tumors with negative surgical margins, MMP-9 expression was significantly higher in samples from patients with biochemical recurrence of the disease. Therefore, this study yielded additional insight into the potential value of the biomarkers investigated here, particularly, the utility of MMP-2 and MMP-9 expression for predicting the invasive behavior of the tumor and justification for therapy. This utility has been highlighted by numerous works that have explored the expression of MMP-2 and MMP-9 in serum, urine, and tissues from prostate cancers. Sauer and colleagues linked higher expression of MMP-2 and MMP-9 in serum and tissues with higher Gleason scores [34]. In our study, MMP-2 and MMP-9 were expressed in tumors with poor differentiation and higher Gleason scores, but there were no significant differences between low Gleason score $(<7)$ and high-grade cancer (Gleason score $>7$ ). These enzymes also displayed significantly higher values in the serum of metastatic carcinoma. In patients with pT3 tumors, increased expression of MMP-2 and MMP-9 was associated with shorter disease-free survival periods; the expression levels of these markers exhibited statistical significance in predicting recurrence. Significant decreases in the levels of these two markers in the sera of patients with metastatic disease after therapy are useful as independent predictors of disease stage in combination with the expression of adhesion molecules [35].

\section{Conclusion}

The results of this study underscore the potential value of the expression levels of MMP-2 and MMP-9 for predicting the invasive behavior of tumors with positive surgical margins; further investigation of this observation and its utility is warranted. In our patients with tumors with negative surgical margins, increased MMP-9 expression above the cut-off value was a good predictor of biochemical recurrence, whereas MMP-2 expression was associated with an increased risk of relapse.

\section{Conflict of Interests}

The authors declare that they have no competing interests.

\section{Acknowledgments}

The authors thank their technicians Ozren Štanfel and Ankica Brajenić for technical assistance and Professor of Mathematics Ivan Dražić for statistical analysis. This work was supported by the Ministry of Science, Education, and Sports of the Republic of Croatia, Grant no. 062-0620095-0077.

\section{References}

[1] S. Valastyan and R. A. Weinberg, "Tumor metastasis: molecular insights and evolving paradigms," Cell, vol. 147, no. 2, pp. 275292, 2011.

[2] J. Westermarck and V. Kähäri, "Regulation of matrix metalloproteinase expression in tumor invasion," FASEB Journal, vol. 13, no. 8, pp. 781-792, 1999.

[3] A. H. Said, J. P. Raufman, and G. Xie, "The role of matrix metalloproteinases in colorectal cancer," Cancers, vol. 6, no. 1, pp. 366-375, 2014. 
[4] A. Talvensaari-Mattila, P. Pääkkö, M. Höyhtyä, G. BlancoSequeiros, and T. Turpeenniemi-Hujanen, "Matrix metalloproteinase- 2 immunoreactive protein: a marker of aggressiveness in breast carcinoma," Cancer, vol. 83, no. 6, pp. 1153-1162, 1998.

[5] A. Talvensaari-Mattila, P. Pääkkö, and T. Turpeenniemi-Hujanen, "MMP-2 positivity and age less than 40 years increases the risk for recurrence in premenopausal patients with nodepositive breast carcinoma," Breast Cancer Research and Treatment, vol. 58, no. 3, pp. 287-293, 1999.

[6] A. Talvensaari-Mattila, P. Pääkkö, G. Blanco-Sequeiros, and T. Turpeenniemi-Hujanen, "Matrix metalloproteinase-2 (MMP2 ) is associated with the risk for a relapse in postmenopausal patients with node-positive breast carcinoma treated with antiestrogen adjuvant therapy," Breast Cancer Research and Treatment, vol. 65, no. 1, pp. 55-61, 2001.

[7] A. Talvensaari-Mattila, P. Pääkkö, and T. TurpeenniemiHujanen, "Matrix metalloproteinase-2 (MMP-2) is associated with survival in breast carcinoma," British Journal of Cancer, vol. 89, no. 7, pp. 1270-1275, 2003.

[8] A. Sivula, A. Talvensaari-Mattila, J. Lundin et al., "Association of cyclooxygenase- 2 and matrix metalloproteinase-2 expression in human breast cancer," Breast Cancer Research and Treatment, vol. 89, no. 3, pp. 215-220, 2005.

[9] A. Väisänen, M. Kallioinen, P. J. Taskinen, and T. Turpeenniemi-Hujanen, "Prognostic value of MMP-2 immunoreactive protein $(72 \mathrm{kD}$ type IV collagenase) in primary skin melanoma," The Journal of Pathology, vol. 186, no. 1, pp. 51-58, 1998.

[10] A. Väisänen, M. Kallioinen, K. von Dickhoff, L. Laatikainen, M. Höyhtyä, and T. Turpeenniemi-Hujanen, "Matrix metalloproteinase-2 (MMP-2) immunoreactive protein-a new prognostic marker in uveal melanoma?" The Journal of Pathology, vol. 188, no. 1, pp. 56-62, 1999.

[11] W. F. Grigioni, A. D'Errico, C. Fortunato et al., "Prognosis of gastric carcinoma revealed by interactions between tumor cells and basement membrane," Modern Pathology, vol. 7, no. 2, pp. 220-225, 1994.

[12] P. Ring, K. Johansson, M. Höyhtyä, K. Rubin, and G. Lindmark, "Expression of tissue inhibitor of metalloproteinases TIMP-2 in human colorectal cancer-a predictor of tumour stage," British Journal of Cancer, vol. 76, no. 6, pp. 805-811, 1997.

[13] A. Juuti, J. Lundin, S. Nordling, J. Louhimo, and C. Haglund, "Epithelial MMP-2 expression correlates with worse prognosis in pancreatic cancer," Oncology, vol. 71, no. 1-2, pp. 61-68, 2007.

[14] D. Trudel, Y. Fradet, F. Meyer, F. Harel, and B. Têtu, "Significance of MMP-2 expression in prostate cancer: an immunohistochemical study," Cancer Research, vol. 63, no. 23, pp. 85118515, 2003.

[15] P. Kuvaja, A. Talvensaari-Mattila, P. Pääkkö, and T. Turpeenniemi-Hujanen, "Low serum level of pro-matrix metalloproteinase 2 correlates with aggressive behavior in breast carcinoma," Human Pathology, vol. 37, no. 10, pp. 1316-1323, 2006.

[16] O. Kuittinen, Y. Soini, and T. Turpeenniemi-Hujanen, "Diverse role of MMP-2 and MMP-9 in the clinicopathological behavior of Hodgkin's lymphoma," European Journal of Haematology, vol. 69, no. 4, pp. 205-212, 2002.

[17] A. Scorilas, A. Karameris, N. Arnogiannaki et al., "Overexpression of matrix-metalloproteinase- 9 in human breast cancer: a potential favourable indicator in node-negative patients," British Journal of Cancer, vol. 84, no. 11, pp. 1488-1496, 2001.
[18] S. M. Ranuncolo, E. Armanasco, C. Cresta, E. B. De Kier Joffe, and L. Puricelli, "Plasma MMP-9 (92 kDa-MMP) activity is useful in the follow-up and in the assessment of prognosis in breast cancer patients," International Journal of Cancer, vol. 106, no. 5, pp. 745-751, 2003.

[19] A. Talvensaari-Mattila, M. Santala, Y. Soini, and T. Turpeenniemi-Hujanen, "Prognostic value of matrix metalloproteinase2 (MMP-2) expression in endometrial endometrioid adenocarcinoma," Anticancer Research B, vol. 25, no. 6, pp. 4101-4105, 2005.

[20] K. W. Kwak, H. M. Lee, and H. Y. Choi, "Impact of capsular incision on biochemical recurrence after radical perineal prostatectomy," Prostate Cancer and Prostatic Diseases, vol. 13, no. 1, pp. 28-33, 2010.

[21] D. Cao, A. S. Kibel, F. Gao, Y. Tao, and P. A. Humphrey, "The gleason score of tumor at the margin in radical prostatectomy is predictive of biochemical recurrence," American Journal of Surgical Pathology, vol. 34, no. 7, pp. 994-1001, 2010.

[22] A. Heidenreich, J. Bellmunt, M. Bolla et al., "Guidelines on prostate cancer," European Urology, vol. 59, no. 1, pp. 572-583, 2011.

[23] K. W. Kwak, H. M. Lee, and H. Y. Choi, "Impact of capsular incision on biochemical recurrence after radical perineal prostatectomy," Prostate Cancer and Prostatic Diseases, vol. 13, no. 1, pp. 28-33, 2010.

[24] W. E. Grizzle, D. G. Bostwick, H. Burke et al., "Biomarkers in prostate cancer," in AACR Education Book, pp. 196-204, Intenational Union Against Cancer, Washington, DC, USA, 2005.

[25] D. M. Somford, I. M. van Oort, J. Cosyns, J. A. Witjes, L. A. L. M. Kiemeney, and B. Tombal, "Prognostic relevance of number and bilaterality of positive surgical margins after radical prostatectomy," World Journal of Urology, vol. 30, no. 1, pp. 105$110,2012$.

[26] R. Thurairaja, J. Osborn, J. Mcfarlane, A. Bahl, and R. Persad, "Radical prostatectomy with positive surgical margins: how are patients managed?" BJU International, vol. 97, no. 3, pp. 445450, 2006.

[27] S. S. Chang and M. S. Cookson, "Impact of positive surgical margins after radical prostatectomy," Urology, vol. 68, no. 2, pp. 249-252, 2006.

[28] Y. Yang, "Treatment of the positive surgical margin following radical prostatectomy," Chinese Medical Journal, vol. 121, no. 4, pp. 375-379, 2008.

[29] D. G. Bostwick, D. J. Grignon, M. E. H. Hammond et al., "Prognostic factors in prostate cancer: college of American pathologists consensus statement 1999," Archives of Pathology and Laboratory Medicine, vol. 124, no. 7, pp. 995-1000, 2000.

[30] D. Cao, A. S. Kibel, F. Gao, Y. Tao, and P. A. Humphrey, "The gleason score of tumor at the margin in radical prostatectomy is predictive of biochemical recurrence," American Journal of Surgical Pathology, vol. 34, no. 7, pp. 994-1001, 2010.

[31] E. Llano, A. M. Pendas, P. Aza-Blanc, T. B. Kornberg, and C. Lopez-Otin, "Dm1-MMP, a matrix metalloproteinase from Drosophila with a potential role in extracellular matrix remodeling during neural development," Journal of Biological Chemistry, vol. 275, no. 46, pp. 35978-35985, 2000.

[32] G. Klein, E. Vellenga, M. W. Fraaije, W. A. Kamps, and E. S. J. M. De Bont, "The possible role of matrix metalloproteinase (MMP)-2 and MMP-9 in cancer, e.g. acute leukemia," Critical Reviews in Oncology/Hematology, vol. 50, no. 2, pp. 87-100, 2004. 
[33] A. Di Carlo, A. Mariano, D. Terracciano et al., "Matrix metalloproteinase-2 and -9 in the urine of prostate cancer patients," Oncology Reports, vol. 24, no. 1, pp. 3-8, 2010.

[34] C. G. Sauer, A. Kappeler, M. Späth et al., "Expression and activity of matrix metalloproteinases- 2 and -9 in serum, core needle biopsies and tissue specimens of prostate cancer patients," Virchows Archiv, vol. 444, no. 6, pp. 518-526, 2004.

[35] R. Roy, J. Yang, and M. A. Moses, "Matrix metalloproteinases as novel biomarkers and potential therapeutic targets in human cancer," Journal of Clinical Oncology, vol. 27, no. 31, pp. 52875297, 2009. 


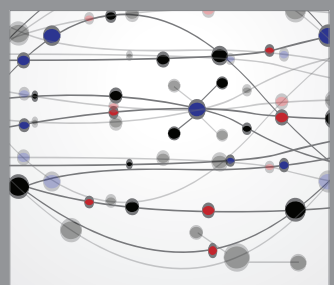

The Scientific World Journal
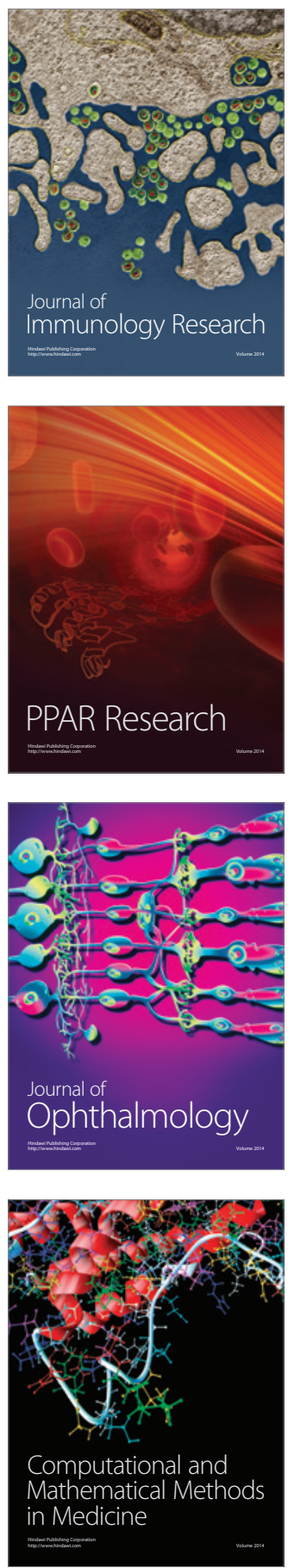

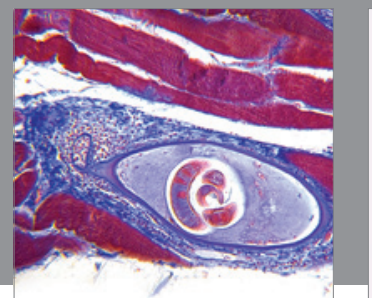

Gastroenterology

Research and Practice
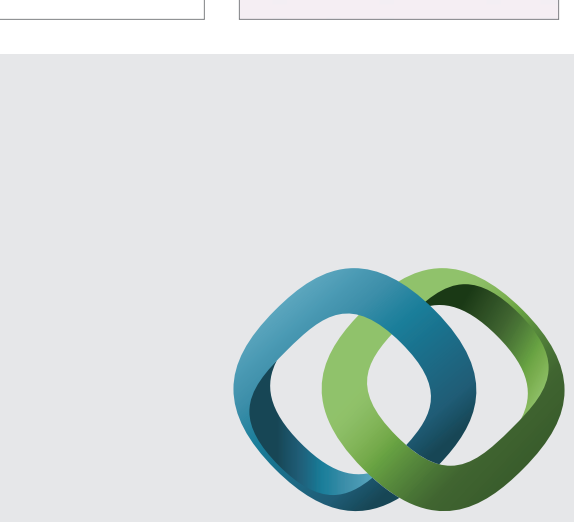

\section{Hindawi}

Submit your manuscripts at

http://www.hindawi.com
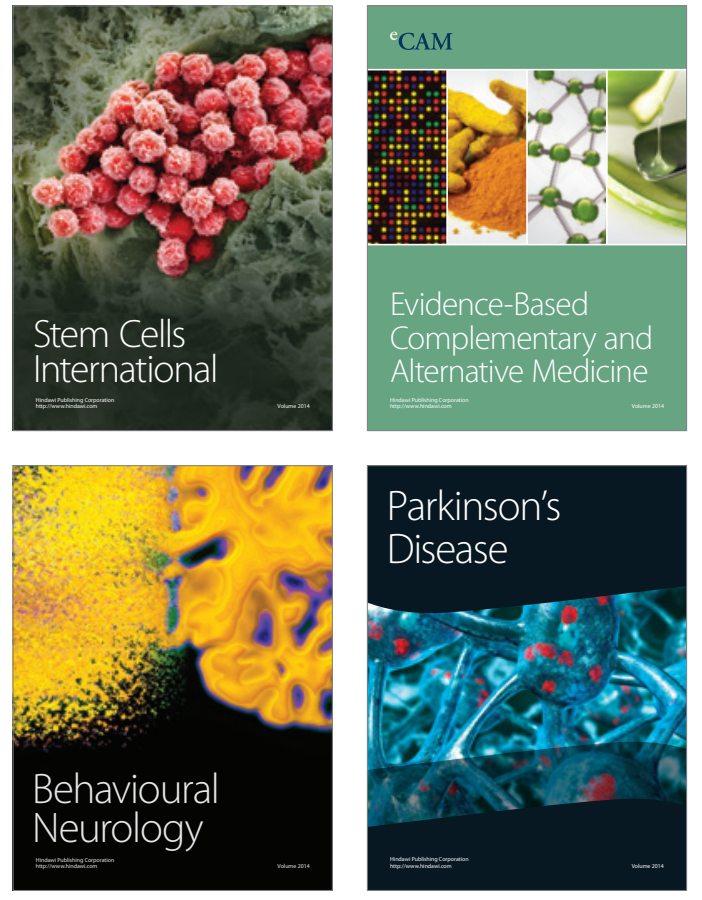
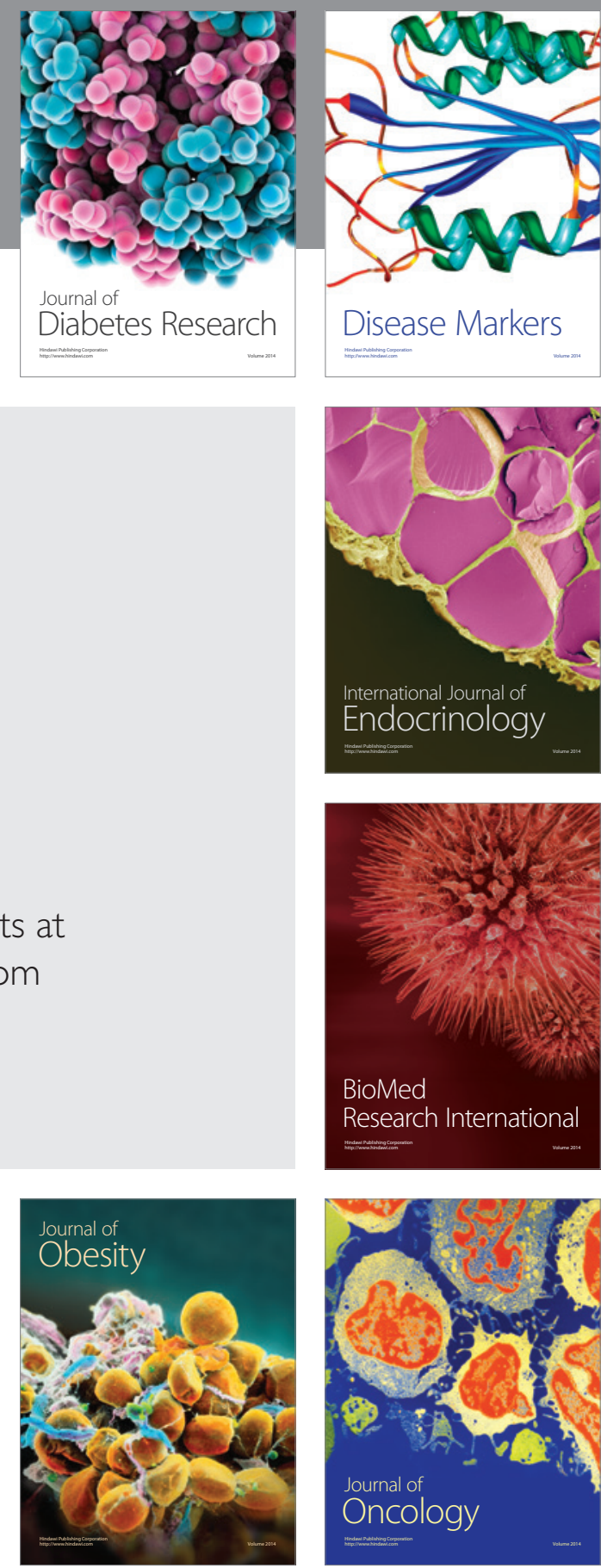

Disease Markers
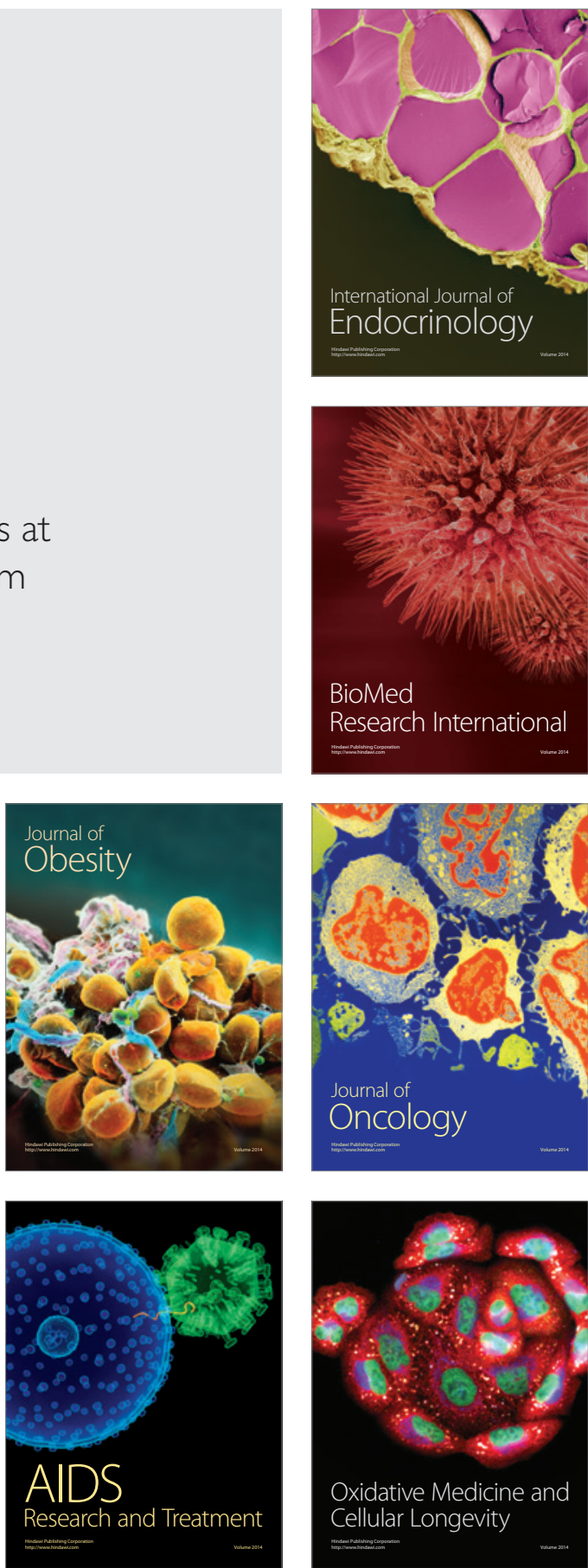